\title{
Intermédialités
}

Histoire et théorie des arts, des lettres et des techniques

Intermediality

History and Theory of the Arts, Literature and Technologies

\section{Le travail de la viande}

\section{Johanne Lamoureux}

Numéro 11, printemps 2008

Travailler (Harun Farocki)

Working (Harun Farocki)

URI : https://id.erudit.org/iderudit/037535ar

Aller au sommaire du numéro

Éditeur(s)

Centre de recherche sur l'intermédialité

ISSN

1705-8546 (imprimé)

1920-3136 (numérique)

Découvrir la revue

Citer cet article

Lamoureux, J. (2008). Le travail de la viande. Intermédialités / Intermediality, (11), 13-34.
Résumé de l'article

Le présent article s'attarde au rôle significatif dévolu, durant le premier tiers du $20^{\mathrm{e}}$ siècle, à la représentation culturelle des abattoirs comme emblème de la modernité industrielle. Il met toutefois moins l'accent sur la performance sacrificielle, telle que la commente le projet contemporain de Georges Bataille, que sur la scénographie machinique qui organise en Amérique la rencontre du travailleur et de l'animal et qui semble avoir fasciné romanciers, dramaturges, cinéastes et dessinateurs de cette période. On s’emploiera à traquer deux topoï exemplaires : le premier a trait à l'animalisation du travailleur et le second à son effacement, à sa dévoration par la machine qui se nourrit de lui et le transforme en viande. 


\title{
Le travail de la viande
}

\author{
Johanne Lamoureux
}

Killing itself then cannot be mechanized. It is upon organization that the burden falls. In one of the great packing plants, an average of two animals are killed every second-a daily quota of some 60,000 head. The death cries of the animals whose jugular veins have been opened are confused with the rumbling of the great drum, the whirring of gears, and the shrilling sound of steam. Death cries and mechanical noises are almost impossible to disentangle. Neither can the eye quite take in what it sees. On one side of the sticker are the living; on the other side, the slaughtered. Each animal hangs head downwards at the same regular interval, except that, from the creatures to his right, blood is spurting out of the neck-wound in the tempo of the heart beat. In twenty seconds, on the average, a hog is supposed to have bled to death. It happens so quickly, and is so smooth a part of the production process, that emotion is barely stirred.

What is truly starling in this mass transition from life to death is the complete neutrality of the act. One does not experience, one does not feel; one merely observes. It may be that nerves that we do not control rebel somewhere in the subconscious. Days later, the inhaled odor of blood suddenly rises from the walls of one's stomach, although no trace of it can have clung to the person.

How far the question is justified we do not know, nevertheless it may be asked: has this neutrality toward death had any further effect upon us? This broader influence does not have to appear in the land that evolved mechanized killing, or even at the time the methods came about. This neutrality toward death may be lodged deep in the roots of our time. It did not bare itself on a large scale until the War, when whole population, as defenceless as the animals hooked head downwards on the traveling chain, were obliterated with trained neutrality. ${ }^{1}$

1. Siegfried Giedion, Mechanization Takes Command. A Contribution to Anonymous History, New York, Oxford University Press, 1948, p. 246. Paru en français sous le titre de La mécanisation au pouvoir. Contribution à l'histoire anonyme, trad. Paule Guivarch, Paris, Centre Georges Pompidou/Centre de Création industrielle, 1980. 
Siegfried Giedion conclut en ces termes le chapitre nodal de Mechanization Takes Command qu'il consacre à la «mécanisation de la mort». L'émotion contenue de cette conclusion suspend exceptionnellement le ton neutre associé à la production du discours scientifique, ton auquel souscrit le reste de l'ouvrage. Giedion y décrit les découvertes et les brevets qui ont rendu possible la série d'opérations par lesquelles la raison technique a su littéralement orchestrer la mise en boîte du vivant. Pourtant, avant même cette conclusion, le ton neutre de l'auteur est contrebalancé par un script visuel qui paraît fondé sur une radicalisation critique de la double projection d'images et de la règle du «compare and contrast» qui, dans la foulée des méthodes pédagogiques d'Heinrich Wölfflin (1864-1945), firent les beaux jours de l'institutionnalisation universitaire de l'histoire de l'art dans le monde anglo-saxon (Giedion avait étudié auprès de Wölfflin dont il occupa la chaire à l'Université de Bâle: le sous-titre de son ouvrage, «contribution à l'histoire anonyme", n'est pas sans rappeler le projet wölfflinien d'une «histoire de l'art sans noms»)2. Une des doubles pages de ce script (fig. 1) oppose deux représentations de bovidés: celle de gauche illustre la bonne utilisation du pistolet avec lequel le bétail est assommé au début de la chaîne de travail, alors que sur la page de droite, une publicité des Meatpackers de Chicago montre un bovin souriant et enguirlandé, image typique qui circule des anciennes représentations de fêtes foraines à la rhétorique publicitaire contemporaine, et qui joue de «l'assentiment de l'animal au fait d'être mangés».

Depuis la parution de l'ouvrage de Giedion, les abattoirs et la consommation de viande ont été appropriés par divers discours militants en tant que prémices et prémisses de la solution finale ou de l'exploitation sexuelle des femmes ${ }^{4}$ :

2. À propos de «l'histoire sans noms» de Wölfflin, voir Arnold Hauser, «The Philosophical Implications of Art History: Art History Without Names», in The Philosophy of Art History, Evanston, Northwestern University Press, 1985, p. 117-276; Heinrich Dilly, «Heinrich Wölfflin: Histoire de l'art et germanistique entre 1910 et 1925 », Revue germanique internationale, $\mathrm{n}^{\circ}$ 2, 1994. Pour une indispensable réfutation des propos de Dilly sur le projet non héroïque de l'histoire de l'art sans noms, on lira les pages qu'Éric Michaud consacre au caractère raciste et nationaliste de l'histoire de l'art sans noms dans Histoire de l'art: une discipline à ses frontières, Paris, Hazan, 2004, p. 80-84.

3. Florence Burgat, «Défiguration et reconfiguration des animaux dans la présentation des viandes et dans l'imagerie publicitaire », Revue d'esthétique, n 40, 2001, p. 57-69.

4. Voir par exemple Carol J. Adams, The Sexual Politics of Meat. A Feminist-Vegetarian Critical Theory, New York, Continuum, 1994 et The Pornography of Meat, New York et Londres, Continuum, 2003 ou Charles Patterson, Eternal Treblinkla: Our Treatment of Animals and the Holocaust, New York, Lantern Books, 2002. Ce dernier titre est disponible en français: Un éternel Treblinka, trad. Dominique Letellier, Paris, Calmann-Lévy, 2007. 
il y aurait là un chapitre intéressant à écrire sur les rapports de la modernité à la violence, tels que les investit désormais le discours éthique sur la consommation de chair animale. Pourtant, mon propos s'inscrit plutôt en amont de la parution de Mechanization Takes Command afin d'explorer le rôle significatif dévolu à la représentation culturelle (roman, film, théâtre) des abattoirs dans la première moitié du $20^{\mathrm{e}}$ siècle. J'écarterai délibérément les écrits et images produits dans la mouvance surréaliste ou péri-surréaliste, notamment autour de Georges Batailles, et m’attarderai à la production culturelle motivée plus directement par le projet socialiste ou marxiste. Moins préoccupée par la performance sacrificielle que par une scénographie machinique qui détermine, à des registres certes différents, une découpe de l'homme comme de l'animal, cette production installe l'abattoir comme site infernal de la modernité industrielle du capitalisme.

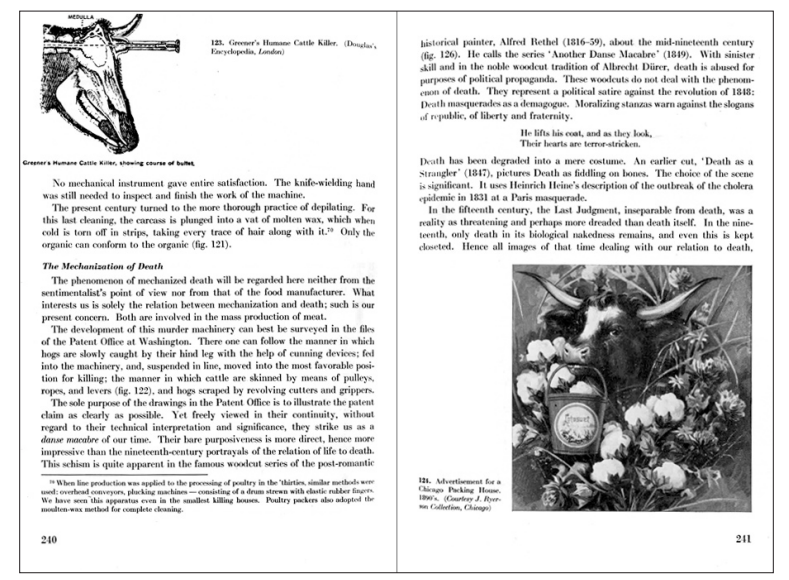

Fig. 1. Siegfried Giedion, «The Mechanization of Death» dans Mechanization Takes Command, Oxford University Press, 1948, p. 240-241.

5. Il est intéressant de rappeler ici que Dawn Ades et Fiona Bradley ont récemment suggéré que le texte de Bataille sur l'abattoir et les photos d'Eli Lotar l'ayant illustré dans la revue Documents $\left(n^{\circ} 6,1929\right)$ auraient été une réaction à la parution, l'année précédente, d'une présentation des Abattoirs de La Mouche à Lyon dans la revue Cahiers d'art $\left(n^{\circ} 8,1928\right)$. Cette présentation préfacée par Christian Zervos de même que les illustrations de cet article insistent sur le caractère fonctionnel (souscrivant aux exigences modernes de l'économie et de l'hygiène) du complexe réalisé par l'architecte Tony Garnier. C'est précisément cette «religion de l'hygiène » que dénonce l'article de Bataille et que nient les photos sanglantes d'Eli Lotar. Voir Dawn Ades et Simon Baker, Undercover Surrealism. Georges Bataille and Documents, catalogue d'exposition de la Hayward Gallery - du 11 mai au 30 juillet 2006, Cambridge (Mass.) et Londres, MIT Press et Hayward Gallery, 2006, p. 14. 
Son récit fondateur se trouve, comme le suggère Giedion, dans The Jungle le roman best-seller de l'écrivain américain Upton Beall Sinclair. Sinclair voit dans l'abattoir la figure emblématique du grand capital et ce, bien avant que Henry Ford ne relate, dans son autobiographie de 1922, le moment épiphanique qu'il connut en visitant les usines à viande de Chicago où il eut l'idée de la chaîne de montage dont on connaît la fortune ultérieure?. Giedion démontre on ne peut plus clairement comment les chaînes de montage (assembly line) de Denver trouvent leur origine dans les chaînes de démontage (disassembly line) de Chicago et comment la mécanisation de la production commence moins dans l'assemblage que dans le démembrement. Les voitures de Ford, assemblées tout au long de la chaîne de montage, sont en quelque sorte les remplaçants machiniques du matériau organique que démonte le travail à la chaîne des abattoirs. Et les abattoirs de Chicago apparaissent, il me semble, à beaucoup d'artistes engagés de l'entre-deux-guerres, comme un modèle plus prégnant de la production industrielle que ne le fut la construction automobile, dans la mesure où il organise, par «le sang des bêtes", une double réification et un double morcèlement du vivant: ceux de la force de travail de l'ouvrier et du corps de la bête qui sert de matière première. Chez Sinclair, mais aussi chez Brecht, Eisenstein ou Chaplin, on voit s'installer autour de la chaîne de montage certains leitmotive du travail automatisé, de son sous-texte sanglant, qui se répètent jusque dans les pages plus conservatrices du Tintin en Amérique d'Hergé $e^{8}$. Nous retiendrons ici deux topoï: l'animalisation du travailleur et sa dévoration par la machine. Les deux sont mis en place dans le roman de Sinclair et ont probablement été répercutés et adaptés suite aux nombreuses traductions de l'ouvrage et à sa grande popularité au sein, notamment, des mouvements de la gauche ouvrière.

The Jungle raconte les espoirs, la désillusion, la dépossession, l'errance et enfin l'éveil politique de Jurgis, un émigré lithuanien qui débarque à Chicago porté par les promesses du rêve américain. D’abord confiant et fier de sa force de travail exceptionnelle, Jurgis déniche un emploi dans les stockyards, fonde une famille, achète une maison puis en vient à être dépossédé de tout: sa maison, sa femme, sa famille, et même sa propre vitalité lorsqu'un accident de travail le conduit à la rue, puis en prison. Le roman est encadré par deux moments épiphaniques qui suggèrent et diffèrent, révèlent et dénoncent l'identification

6. Upton Beall Sinclair, The Jungle [1906], (postface du Docteur Barry Sears), New York, Signet Classics, 2006.

7. Henry Ford, My Life and Work, New York, Garden City, 1922.

8. Hergé, Tintin en Amérique [1947], Paris, Casterman, 1973. 
du travailleur à la bête, telle qu'elle est tramée par les patrons du trust de la viande. D'entrée de jeu, lors de sa première visite aux abattoirs, accédant à cette "pièce d'où les cochons ne reviennent pas9", Jurgis découvre, fasciné, la bruyante agonie des bêtes prises dans l'engrenage de la grande machinerie d'abattage et il se réjouit «de n'être pas un porc ». Poursuivant son itinéraire, il est aussitôt distrait de cette pensée par l'étonnant spectacle du travail qui s'offre à lui :

The carcass hog was scooped out of the vat by the machinery, and then it fell to the second floor, passing on the way through a wonderful machine with numerous scrapers, which adjusted themselves to the size and shape of the animal, and sent it out at the other end with nearly all of its bristles removed. It was then again strung up by machinery, and sent upon another trolley ride; this time passing between two lines of men, who sat upon a raised platform, each doing a certain single thing to the carcass as it came to him. One scraped the outside of a leg; another scraped the inside of the leg. One with a swift stroke cut the throat; another with two swift strokes severed the head, which fell to the floor and vanished through a hole. Another made a slit down the body; a second opened the body wider; a third with a saw cut the breastbone; a fourth loosened the entrails; a fifth pulled them out - ant they also slid through a hole in the floor. There were men to scrape each side and men to scrape the back; there were men to clean the carcass inside, to trim it and wash it. Looking down this room, one saw, creeping slowly, a line of dangling hogs a hundred yards in length; and for every yard there was a man, working as if a demon were after him. At the end of this hog's progress every inch of the carcass had been gone over several times, and then it was rolled into the chilling room, where it stayed for twenty four hours, and where a stranger might lose himself in a forest of freezing hogs [...] Jurgis went down the line with the rest of the visitors, staring open-mouthed, lost in wonder. He had dressed hogs himself in the forest of Lithuania; but he had never expected to live to see one hog dressed by several hundred men. It was like a wonderful poem to him $[\ldots]^{10}$

9. Upton Beall Sinclair, The Jungle, p. 38. Toutes les citations du texte proviennent de cette édition marquant le centième anniversaire de la parution originale du roman de Sinclair. La couverture de cette édition commémorative, illustrée par une gravure d'abattage pré-industriel, de même que la postface de Barry Sears, auteur connu pour sa défense d'une alimentation carnée et pour sa dénonciation du rôle des lobbyistes céréaliers dans l'établissement et la promotion de la pyramide alimentaire, trahissent bien les craintes de l'éditeur que le roman de Sinclair n'exacerbe encore aujourd'hui, dans le contexte actuel de la concurrence des régimes et de celle des lobbies de l'industrie agro-alimentaire, les réserves des lecteurs/consommateurs envers les «produits» de l'abattoir.

10. Upton Beall Sinclair, The Jungle, p. 40-41. 
Dans l'édifice situé de l'autre côté de la rue, là où l'on abat les bovins, c'est encore la spécialisation des tâches qui retient l'attention, mais cette fois, plutôt que la carcasse, c'est le travailleur qui se déplace le long de la chaîne «each man having his task to do; generally this would consist of only two or three specific cuts, and he would pass down the line of fifteen or twenty carcasses, making these cuts upon each ${ }^{11} »$. Non seulement, comme dans ce qui précède, les trajectoires chorégraphiées de l'ouvrier et de l'animal sont-elles en partie échangeables, mais de plus, la longue description des lieux et de la chaîne montre indissociablement la découpe de l'animal et la fragmentation du travail, en rabattant l'un sur l'autre le morcèlement du corps par lequel l'animal est transformé en viande et le morcèlement de la tâche par lequel se réifient le corps et la force de travail de l'ouvrier. Au terme du roman, alors que Jurgis a depuis longtemps quitté la jungle de l'abattoir et sa dense forêt de carcasses, le sens de ce recoupement s'éclaire pour lui :

To Jurgis, the packers had been equivalent to fate: Ostrinski showed him that they were the Beef Trust. They were a gigantic combination of capital, which had crushed all opposition, and overthrown the laws of the land, and was preying upon the people. Jurgis recollected how, when he had first come to Packingtown, he had stood and watched the hog-killing, and thought how cruel and savage it was, and come away congratulating himself that he was not a hog; now this new acquaintance showed him that a hog was just what he had been-one of the packers' hogs. What they wanted from a hog was all the profits that could be got of him; and that was what they wanted from the workingman $[\ldots]^{12}$

Le premier film d'Eisenstein, Stachka (La grève, 1925), revient sur une telle association. Un ouvrier, injustement accusé de vol par ses patrons, se

11. Upton Beall Sinclair, The Jungle, p. 43-44.

12. Upton Beall Sinclair, The Jungle, p. 361. La suite de cet extrait précise que le consommateur est aussi traité de la même manière. On sait d'ailleurs que le succès de scandale du roman découla moins de l'empathie des lecteurs envers les travailleurs que de leur dégoût envers les conditions de production insalubres de la viande. Sinclair, dans une préface ultérieure, déclara «avoir visé le cœur des lecteurs mais, par accident, avoir plutôt atteint leur estomac». Récemment élu lors de la parution initiale du roman, le président Theodor Roosevelt, adepte des préceptes hygiénistes de Kellog, ordonna des enquêtes qui débouchèrent rapidement sur le Federal Meat Inspection Act (1906) et finalement sur la création de l'agence gouvernementale (FDA) encore responsable aujourd'hui de l'inspection alimentaire en sol étasunien. Voir Upton Beall Sinclair, The Jungle, New York, Viking Press, 1947, p. viII. Gail A. Eisnitz, Slaughterhouse. The Shocking Story of Greed, Neglect and Inhumane Treatment Inside the U.S. Meat Industry, Amherst (N.Y.), Prometheus Books, 1997, p. 21. 
suicide en se pendant à la machinerie de l'usine (une image qui rappelle les cochons suspendus de The Jungle). Ses camarades déclenchent une grève qui sera, dans le dénouement du film, réprimée violemment alors que le montage d'Eisenstein fera alterner les scènes de la répression ouvrière et des images de l'abattage d'un bœuf. Le choc de ce montage a été finement préparé par les nombreuses séquences, souvent idylliques, où figurent divers animaux (bouc, oies, chatons, corbeau, etc.) et surtout par la représentation zoomorphe des quatre espions et délateurs au service des patrons, laquelle établit un bestiaire dans la tradition pluri-séculière des dessins physiognomoniques. Or, précisément dans la foulée de cette tradition, ce tétramorphe procède par condensation (des fondus de l'image qui font apparaître l'agent à partir de son «totem ») et non par le principe du montage alterné de la séquence de répression et d'abattage. Le montage des attractions, parallèlement à la fonction pragmatique de choc qu'il impose aux spectateurs, établit, sur le plan sémantique, une comparaison plutôt qu'une identification avec le monde animal. Le travailleur est traité comme une bête: l'espion ressemble à la bête; il est une bête. L'abattage est artisanal chez Eisenstein et ne résulte pas d'un travail sériel et mécanisé. Le bœuf est un individu comme l'est le cochon sur lequel s'apitoie longuement la narration dans The Jungle avant que Jurgis n'en vienne à se réjouir de sa différence. Il ne s'agit pas d'un abattage sériel de 400 à 500 têtes à l'heure, pour s'en tenir aux chiffres donnés par Sinclair (et qui demeurent assez stables de 1866 à 1948 selon Giedion) ${ }^{13}$. La violence de l'abattage est dissociée par Eisenstein de la mécanisation de la mort mais néanmoins elle demeure, dans le contexte de la grève, liée au contexte de l'usine et au régime de production capitaliste ${ }^{14}$.

13. Siegfried Giedion, Mechanization Takes Command, p. 244. "Only the knife, guided by the human hand, performs the transition from life to death in the desired manner. For this operation craftsmen are needed who combine the precision and skill of a surgeon with the speed of a piece worker. It is established how far and how deep the throat of a hog should be pierced. A false stroke injures the meat product. And it must be done quickly - 500 hogs per hour ». En note, Giedion, citant la source de ses chiffres, (Scientific American: 21 août 1866) ajoute que la cadence, en 1948, est encore plus ou moins la même au moment où il écrit: de 500 à 600 bêtes à l'heure. Pour des statistiques plus contemporaines, voir Gail Eisnitz, Slaughterhouse, chapitre 6. Voir aussi sur les conditions actuelles de l'industrie de la viande, Eric Marcus, Meat Market. Animal, Ethics and Money, Boston, Brio Press, 2005, p. 5-14.

14. Ce n'est d'ailleurs pas la seule référence à l'abattage ou à la viande dans l'œuvre d'Eisenstein. On pensera notamment à Staroye i novoye (La ligne générale, 1929) ou au Bronenosiets Potyomkin (Cuirassé Potemkine, 1925). Dans ce dernier film, la viande pourrie 
Il faut par ailleurs noter que la nouveauté et la modernité de la chaîne de démontage chez Sinclair ne sont jamais exaltées selon l'esthétique machiniste que le futurisme s'apprête alors à glorifier et qui triomphera après la Grande Guerre dans les mouvements dada et constructiviste. Rien ici de ces engrenages et mécanismes qui semblent opérer tout seuls sans produire quoi que ce soit, comme la belle machine «célibataire» du Metropolis de Lang (1926), dont la seule fonction est apparemment de soumettre le corps des travailleurs à une étrange gymnastique bio-mécanique. Au contraire, l'évocation par Sinclair des dispositifs de Packingtown fait l'économie de tout regard esthétique et si elle les représente comme une merveille d'ingéniosité, elle les rapproche aussi des instruments de torture alors que les chambres de tuerie sont apparentées au donjon médiéval. On est donc loin alors de la séduction rutilante de l'usine high tech des Modern Times (Les temps modernes, 1936).

Or il semble à bien y regarder que Chaplin, dans la première partie du film où le vagabond se retrouve à l'usine, n'ait pas oublié le revers d'animalisation du travailleur qui accompagne la fragmentation et l'automatisation de sa tâche. Le film s'ouvre sur l'image d'un troupeau de moutons qui se transforme bientôt en cortège de travailleurs procédant vers l'usine ${ }^{15}$. On retrouve le vagabond en

qui déclenche la révolte des marins du cuirassé constitue une métaphore du capitalisme qui paraît directement inspirée par les textes de Lénine. Sur le caractère «pourrissant» du régime invoqué par ce dernier, notamment dans «Programme militaire de la révolution prolétarienne» (1916), voir Laura Bossi, «L'homme nouveau. Dégénérescence et régénération », p. 37 dans Jean Clair, Les Années 1930. Fabrique de "L'Homme nouveau ", Paris et Ottawa, Gallimard et Musée des Beaux-arts du Canada, 2008, p. 36-57, catalogue éponyme de l'exposition organisée par Pierre Théberge (6 juin-7 septembre 2008).

15. Le rapprochement du bétail et des travailleurs (comme celui du travail et de l'abattoir) relève déjà du lieu commun à cette époque. On le trouve établi très en amont, par exemple chez Zola dans L'assommoir (1877). Dés le début du roman, Gervaise, accoudée à la fenêtre au petit matin, attend, inquiète, le retour de son homme et observe la rue: «à droite, du côté du boulevard de Rochechouart, où des groupes de bouchers, devant les abattoirs, stationnaient en tabliers sanglants; et le vent frais apportait une puanteur par moments, une odeur fauve de bêtes massacrées. Elle regardait à gauche, enfilant un long ruban d'avenue, s'arrêtant, presque en face d'elle, à la masse blanche de l'hôpital de Lariboisière, alors en construction». Zola poursuit en décrivant «le flot ininterrompu d'hommes, de bêtes, de charrettes, qui descendait des hauteurs de Montmartre et de la Chapelle. Il y a avait là un piétinement de troupeau, une foule que de brusques arrêts étalaient en mares sur la chaussée, un défilé sans fin d'ouvriers allant au travail, leurs outils sur le dos, leur pain sous le bras». Émile Zola, L'assommoir dans Euvres complètes, tome II, Paris, Gallimard, Bibliothèque de la Pléiade, p. 376-377. Outre l'encadrement 
travailleur à la chaîne qui essaie de garder la cadence ; à l'heure du déjeuner, il est choisi par le patron pour tester une nouvelle machine qui permet de s'alimenter en réduisant la perte de temps et d'énergie qu'occasionne le repas. Immobilisé et privé de l'usage de ses mains, il subit les assauts de cette machine qui lui sert notamment du maïs, aliment exemplaire de la diète américaine du bétail, et se trouve impuissant lorsque le prototype s'avère dysfonctionnel et se détraquant complètement, le nourrit sans succès et à une cadence frénétique. Au moment où la spécialisation du travail l'affole et l'enferme dans la répétition compulsive du seul geste auquel se réduit sa tâche, le vagabond détourne ses instruments de travail, et dans une appropriation bricoleuse, au sens où Levi-Strauss conçoit le bricolage ${ }^{16}$, il les convertit en cornes, s'en orne le front et charge vers la sortie. Enfin, revenu à l'usine, il termine son escapade en redescendant des passerelles supérieures, suspendu à un immense crochet, comme une carcasse fraîchement équarrie ou comme un quartier de viande.

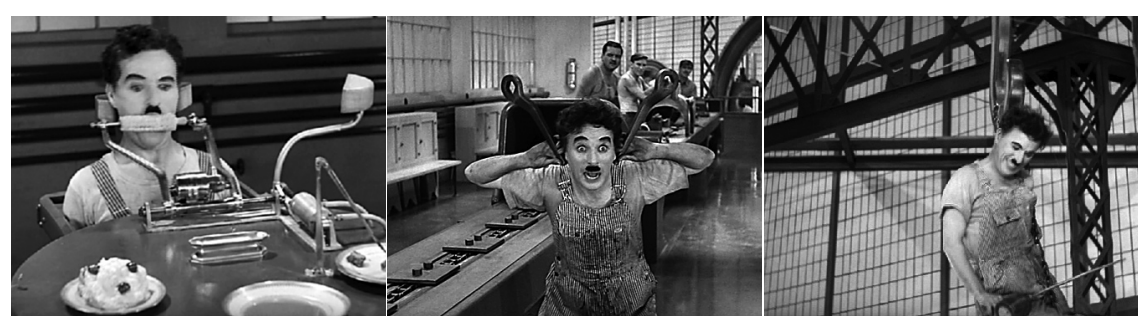

Fig: 2a, 2b, 2c: Charlie Chaplin, Modern Times (Les temps modernes, 1936), (CMK2.

L'animalisation du travailleur, disséminée en quelques images furtives, récapitule les diverses étapes du sort de la bête d'élevage: son alimentation, sa transhumance en troupeau (ce que l'industrie appelle la «viande sur pattes»), le passage de l'organisme dans la machine et l'apparition dernière du corps suspendu au crochet. Elle traduit certes l'aliénation du travailleur, son devenirautre, mais en le déclinant à travers une modalité souvent ignorée au profit de la

topologique de la vie ouvrière déjà mentionné, Zola accorde aussi un rôle déterminant à la viande, comme emblème d'une esthétique matérialiste cette fois, mais cette élaboration se joue moins autour de l'abattoir et du démembrement de la production industrielle qu'à partir du faire vrai, du faire cru, de la composition naturaliste, telle qu'elle se manifeste exemplairement dans l'étalage de viande et de charcuterie que réalise le peintre Claude Lantier dans Le ventre de Paris (1873), dans (Euvres complètes, tome I, p. 80o-801.

16. Claude Levi-Strauss, La pensée sauvage, Paris, Plon, p. 48-53. 
«folie» qui, au terme de cet épisode, conduit le vagabond à l'asile. Pourtant c'est aussi cette animalisation qui organise une première échappée hors de l'usine dans une péripétie qui la rapproche davantage d'un «devenir-animal » : la séquence où, détournant les tenailles avec lesquelles il resserre les boulons et les transforme en cornes, le travailleur s'en coiffe et charge vers l'extérieur, suggère que l'animalisation est ici assumée pour être brouillée et retournée, qu'il y est moins question de ressemblance formelle avec la bête que d'un éloge de sa force, non plus sa force de production mais sa force de fuite ${ }^{17}$.

Il revient à Brecht, dans Sainte Jeanne des Abattoirs (1929-1930) ${ }^{18}$, d'avoir brouillé, en la distribuant à différents points de vue, cette construction identificatoire entre le travailleur et l'animal d'abattage. Daté du 15 avril 1920, un commentaire critique du dramaturge allemand sur une production du Don Carlos de Schiller évoque sa lecture de The Jungle de Sinclair ${ }^{19}$. Mais c'est seulement une dizaine d'années plus tard, alors qu'il a entrepris de lire Marx et qu'il s'emploie à formuler les préceptes de son théâtre épique, que Brecht élabore sa Sainte Jeanne. Or, il est intéressant de noter que dès cette première mention du roman américain, rédigée l'année même de la canonisation de Jeanne d'Arc, Brecht associe déjà le marché de la viande et le drame romantique allemand, genre que sa pièce parodiera, dans un renversement carnavalesque, par ses

17. Sur le devenir-animal, voir Gilles Deleuze et Félix Guattari, Capitalisme et schizophrénie. Tome 2: Mille plateaux, Paris, Les Éditions de Minuit, coll. «Critique», 1980, p. 284-380 et sur la viande et le devenir-animal, Gilles Deleuze, Francis Bacon. Logique de la sensation [1981], Paris, Éditions du Seuil, coll. «L'ordre philosophique», 2002, p. 27-32.

18. Bertolt Brecht, Die Heilige Johanna der Schlachthöfe, Francfort, Suhrkamp, 1962. Texte français de Gilbert Badia avec la collaboration de Claude Duchet: Sainte Jeanne des abattoirs, dans Théâtre complet, vol. 2, Paris, L'Arche, 1974.

19. Bertolt Brecht, Saint Joan of the Stockyards, trad. Frank Jones, Bloomington, Indiana University Press, 1969. L'extrait est cité par Frederic Garb dans son introduction à cette édition, p. 9: «Ces jours-ci, j’ai lu La jungle de Sinclair, l'histoire d'un travailleur qui meurt de faim aux abattoirs de Chicago. Il y est question de la faim, du froid, de la maladie qui achèvent un homme aussi sûrement que s'ils venaient de Dieu. Cet homme entrevoit une petite vision de la liberté, mais il est bientôt abattu à coups de matraque. Je sais que sa liberté n’a rien à voir du tout avec Carlos - mais je ne peux plus considérer sérieusement la servitude de Carlos» (notre traduction). Évoquant le retentissement international de son roman et particulièrement sa réception allemande, Upton Beall Sinclair remarque que The Jungle fut probablement «le seul livre promu à la fois par les socialistes et les junkers, ces derniers cherchant à obtenir de la viande importée à meilleur prix». Upton Beall Sinclair, The Jungle, 1947, p. x. 
chœurs en vers libres et par de nombreuses citations (à la Pucelle d'Orléans de Schiller mais aussi au Faust II de Goethe) ${ }^{20}$.

20. Évoquant le lien qu'établit Brecht entre Sinclair et Schiller dans son commentaire de 1920, Frederic Garb note: "Here already is the antithesis between the idealism of German classical drama and the stark realities of life in the big city which form a basic structural element of Saint Joan of the Stockyards». Bertolt Brecht, Saint Joan of the Stockyards, p. 9. Le lecteur pourra comparer les passages de Schiller et de Goethe cités par Brecht aux reprises parodiques qu'il en produit dans Sainte Jeanne en consultant Brechts Die Heilige Johanna, textes réunis et présentés par Jan Knopf, Francfort, Surhkamp, coll. «Suhrkamp Taschenbuch Materialien», 1986. Rainer Fassbinder s'est peut-être rappelé ce détournement du drame allemand puisque la célèbre scène des abattoirs de In einem Jahr mit 13 Monden (L'année des treize lunes, 1978) se livre au même exercice: Elvira, l'ex-boucher devenue femme désœuvrée et abandonnée, termine sa visite aux abattoirs en moquant les ambitions d'acteur de son amant Christoph et en déclamant, sur un ton qui se fait graduellement strident, une des dernières tirades du Torquato Tasso de Goethe (Acte $\mathrm{V}$, scène 5). Les plaintes d'un Tasse, figure d'artiste paranoïaque qui s'imagine qu'on lui vole son œuvre et se considère conduit à l'autel du sacrifice, contrastent insupportablement avec le silence des bœufs qu'on voit au premier plan être assommés, égorgés et saignés. Voir la traduction, malheureusement vieillie, d'Hippolyte Loiseau reprise dans l'édition bilingue de Torquato Tasso, Paris, Aubier-Montaigne, coll. «Bilingue», 1977, p. 106-110. Les renversements sont fréquents chez Fassbinder et Thomas Elsaesser a analysé, autour précisément de L'année des treize lunes, le renversement sociopolitique que le cinéaste construit autour de la figure d'Anton, le survivant juif des camps devenu malgré lui le bourreau sentimental d'Elvira qui a changé de sexe pour lui sans en être aimé en retour. Voir Thomas Elsaesser, R.W. Fassbinder, un cinéaste d'Allemagne, trad. Christophe Jouanlanne, Pierre Rusch et Jean Torrent, Paris, Centre Georges Pompidou, 2005, p. 324-353. Le motif de la viande, de la production de viande, donnerait donc lieu chez Brecht et chez Fassbinder à un détournement parodique qui relève du carnaval, et ce, non plus seulement au travers de la seule étymologie du terme suggérée par son radical carne. Ce qui est en jeu dans ce recours parodique, c'est certes la mise à distance (du spectateur chez Brecht et du spectacle chez Fassbinder) mais c'est aussi le principe carnavalesque du renversement formulé par Bakhtine. Le motif de la viande, encore investi d'une valeur de choc qui semble ne jamais devoir s'émousser, déclenche et supporte une opération de subversion du canon et de la litanie que les étals de viande de la peinture de genre amorcent d'une certaine manière dès la seconde moitié du $16^{e}$ siècle. Voir sur cette période l'article de Sheila Mc Tighe, «Foods and the Body in Italian Genre Paintings, about 1580: Campi, Passarotti, Carracci », Art Bulletin, volume 86, n², juin 2004. Enfin, Michael F. Zimmerman a récemment rapproché les «tensions» d'un travail sur l'histoire et sur la chair qui marqueraient à la fois l'œuvre de Lovis Corinth et celui de Rainer W. Fassbinder: "Corinth et la chair de la peinture» dans Lovis Corinth 1858-1925 Entre impressionnisme et expressionnisme, Serge Lemoyne et Marie-Amélie zu Salm-Salm (dir.), 
Sainte Jeanne des Abattoirs retrace l'éveil politique de Jeanne Dark, une militante chrétienne de l'Armée du Salut, exposée aux stratagèmes d'un baron de l'industrie de la viande, Pierpont Mauler, dont le nom évoque, par le prénom et les initiales, le "philanthrope » Pierpont Morgan, et par le patronyme (Maul: mâchoire et gueule en allemand), la voracité broyante du personnage. Persuadée au début que la véritable action chrétienne se limite à venir en aide aux travailleurs et que la seule façon d'y arriver est de se situer au-dessus de la mêlée et du conflit qui les oppose aux patrons, Jeanne comprend bientôt que cette position équivaut en fait à se ranger du côté des possédants et de la violence qu'ils exercent et elle reconnaît in extremis, au terme d'un cheminement hésitant où elle a trahi les ouvriers, que seule la violence peut répondre à la violence ${ }^{21}$.

Sainte Jeanne est une pièce jumelle de La mère (1929-1930)22, figure que Brecht opposera à Jeanne, femme virginale, au double sens, sexuel et religieux, du

exposition éponyme qui s'est tenue à Paris, Musée d'Orsay, du $1^{\text {er }}$ avril au 22 juin 2008; Leipzig, Museum der bildenden Künste, du 10 juillet au 18 octobre 2008; Ratisbonne, Kunstforum Ostdeutsche Galerie, du 9 novembre 2008 au 15 février 2009, Paris, Musée d'Orsay, Réunion des musées nationaux, 2008, p. 320-331. Zimmerman analyse par ailleurs finement le rôle formateur pour le peintre qu'un récit de Corinth, rédigé en 1909, confère à l'abattoir. Cet apprentissage de la chair qui se trouve opposé par Corinth à la formation académique des beaux-arts appartient à ce que je considère comme un premier paradigme du traitement emblématique de la viande par la production culturelle moderne: il y est moins question du travail et de la production que de la matière et de sa transfiguration par le pouvoir de l'art. En ce sens, ce que Zola avait problématisé avec l'étal de charcuterie, la vitrine-manifeste du naturalisme de Claude Lantier dans Le ventre de Paris, se clôt avec Corinth.

21. Frederic Garb suggère, après Hans Eisler, que cette insistance de la pièce sur la nécessité d'une solution violente trahit davantage l'influence léniniste que celle, généralement attribuée au Brecht de cette époque, d'une lecture récente du Capital de Marx. Frederic Garb dans Bertolt Brecht, Saint Joan of the Stockyards, p. 15.

22. Brecht avait aussi envisagé des destinations, des destinataires et des styles de jeu différents pour ses deux pièces. Sur cette question, voir Camille Demange, Bertolt Brecht, Paris, Seghers, coll. «Théâtre de tous les temps », 1967, p. 69-73. «Les deux œuvres qui sont assurément jumelles ont été créées à l'intention de publics différents, La mère pour les organisations féminines prolétariennes et Sainte Jeanne des abattoirs pour les grandes salles où l'on a l'habitude de jouer les classiques.» De La mère, Demange écrit que: "C'est du théâtre de propagande, mais qui se différencie du théâtre de Piscator et de l'agit-prop sur un point essentiel, en ce qu'il ne convie pas seulement le prolétariat à l'action, mais tout autant, et parallèlement, à un effort de culture générale et de culture marxiste. Tout autre était le public qui aurait dû voir Sainte Jeanne des abattoirs si les représentations n'avaient pas été interdites. [...] Brecht voulait que Sainte Jeanne des 
terme : affligée de la stérilité de la pucelle et motivée par un projet d'intercession, celui attribué à la Vierge chrétienne, Jeanne s'avère, selon l'expression même de son patron Snyder, une «médiatrice inutile ${ }^{23}$ », c'est-à-dire une médiatrice dans un contexte où toute médiation, aux yeux de Brecht, se trouve vouée à l'échec et au renforcement de l'exploitation des prolétaires. Frappée de pneumonie, agonisante, Jeanne, dans une dernière scène qui reprend la finale du Faust II, meurt en essayant de faire connaître sa nouvelle position tandis qu'un chœur de bouchers, parlant «le langage des rois» l'encense dans une «humiliante apothéose ${ }^{24}$ » et censure ses propos par l'éloge et le bruit.

Dans une paraphrase de la célèbre métaphore shakespearienne, Jeanne décrit le monde non plus comme un théâtre mais comme un «immense abattoir où nous appelle la rumeur d'imminentes violences ${ }^{25} \gg$. Lusine à viande figure donc chez Brecht ce milieu exemplaire de la violence de classe en milieu urbain: elle le figure d'ailleurs bien davantage qu'elle ne l'incarne car ce qui intéresse Brecht, au premier chef, tient moins au processus de la mécanisation du travail, dans la transformation «à chaque heure de quatre ou cinq cents têtes de bétail en viande ${ }^{26}$ » que dans les opérations abstraites qui fondent la circulation et les métamorphoses du capital. Ce sont les causes et les effets concrets de cette violence que Brecht vise à montrer sur scène. Son sujet est donc moins la viande que l'argent et la nature des transmutations quasi alchimiques du capitalisme. À propos de Mauler, l'homme faustien, le fabricant de conserves

abattoirs figurât au répertoire des classiques à côté de La Pucelle d'Orléans. Pour y apparaître comme la contestation de la tragédie schillerienne, sans doute, mais pas uniquement pour cela. Sainte Jeanne des abattoirs est une tragédie et pas seulement une parodie de tragédie. [...] Ce n’en est pas moins une pièce politique et de culture politique, mais elle appartient à la tradition du théâtre de critique sociale qui met en cause le public auquel elle s'adresse. Il n'est donc pas surprenant que les styles de jeu des deux œuvres soient exactement opposés, alors que leur structure respective procède des mêmes principes du théâtre épique. Schématiquement, on peut dire que La mère est la seule pièce de Brecht qui ne puisse être jouée que dans un style rigoureusement brechtien et que Sainte Jeanne des abattoirs est la seule pièce qui ne puisse être jouée dans le style traditionnel du Berliner Ensemble.» Sainte Jeanne des abattoirs fit l'objet d'une lecture radiophonique en 1932 mais la première représentation sur scène n'eut pas lieu avant 1959 à Hambourg.

23. Bertolt Brecht, Sainte Jeanne des abattoirs, p. 59.

24. Les formules sont de Camille Demange, Bertolt Brecht, p. 70 et p. 67.

25. Bertolt Brecht, Sainte Jeanne des abattoirs, p. 11.

26. Upton Beall Sinclair, The Jungle, p. 43. 
Graham s'exclame: «Il fait de l'or avec de la viande pourrie, et si tu lui jetais à la tête des pierres, il muerait en argent ces pierres elles-mêmes ${ }^{27}$."

La pièce de Brecht ne s'attarde donc pas beaucoup à la description du travail et celle-ci, quand elle surgit, paraît fort inspirée des pages de Sinclair, comme dans cette répartie de Cridle, l'associé de Mauler:

Le nouveau procédé est fort ingénieux.

Le porc est amené sur un tapis roulant

Jusqu'en haut de la chaîne au tout dernier étage.

Le dépeçage alors va commencer pour lui.

Sur les couteaux, tout seul, le porc se précipite.

Bien trouvé, n'est-ce pas? Il s'égorge et bientôt,

Tout seul, saucisse il deviendra,

Car tombant d'étage en étage,

D'abord, il perd sa peau, qui va donner du cuir,

Abandonne ses soies, dont on fera des brosses,

Et sa carcasse enfin, réduite en poudre d'os.

Et ainsi de lui-même par son poids, de lui-même, il arrive

Dans la boîte en fer blanc qui l'attendait en bas.

Belle invention, tu ne trouves pas ${ }^{28}$ ?

Mais alors qu'il faut près de 400 pages au protagoniste de Sinclair avant qu'il ne soit éclairé sur le fait que l'industrie le considère précisément comme le porc qu'il s'était jadis brièvement réjoui de ne pas être, la pièce de Brecht s'ouvre sur l'apitoiement de Mauler au souvenir d'un taureau, «blond et noble » «recevant le coup qui l'abattait». Or il s'agit là d'un travestissement empathique, car Mauler (ainsi qu'un aparté permet au spectateur de le comprendre d'entrée de jeu) a été prévenu par la lettre d'un ami new-yorkais d'une saturation du marché de la viande et il cherche un prétexte pour se défaire de ses parts. Cette ruse d'apitoiement, maintes fois réitérée au fil de la pièce, Brecht la met en contradiction avec toute une série d'autres observations: le bifteck saignant avec lequel Mauler reprend des forces, l'absence de pitié qu'il manifeste envers ses ouvriers quand il dit à Jeanne :

Mais vous prenez bien trop à cœur le sort des pauvres;

Ils sont mauvais. L'homme d'ailleurs ne m'émeut pas.

Aucun n'est innocent. Ce sont tous des bouchers.

$[\ldots]$

J'ai grand pitié des bœufs: l'homme, lui, est mauvais.

27. Bertolt Brecht, Sainte Jeanne des abattoirs, p. 18.

28. Bertolt Brecht, Sainte Jeanne des abattoirs, p. 19. 
$[\ldots]$

À Slift, son courtier :

Emmène-la aux abattoirs et montre-lui

Comme ses pauvres sont mauvais et lâches

Semblables à la bête, et remplis de traîtrise,

Responsables de leur misère ${ }^{29}$.

Les ouvriers, eux, s'indignent de ce que les patrons les considèrent « comme des bœufs prêts à tout ${ }^{3 \circ}$ » et les fabricants de conserves, acculés à la faillite par les spéculations de Mauler, l'invectivent: «Sale boucher. Prends plutôt notre chair. Taille dedans ${ }^{31}$. » Ainsi, à propos des effets de la mécanisation et de la chaîne de montage sur le travailleur, sur l'organisation du travail, sur le travail même de la viande, Brecht, compte tenu de l'angle qu'il adopte, n'a pas autant à dire que Sinclair. Et sa description, déjà citée ci-dessus, du parcours d'un porc à travers la chaîne de l'usine, contraste singulièrement avec le parallèle suggéré par celle de Sinclair : les ouvriers, plutôt que de se trouver réduits à une tâche spécialisée qui les morcèle et les fragmente, redoublant de la sorte les opérations auxquelles ils soumettent les bêtes, ont tout simplement été éliminés dans le passage de Brecht: «Sur les couteaux, tout seul, le porc se précipite.»; «Il s'égorge et bientôt, tout seul, saucisse, il deviendra.» On pourra certes arguer que cette description ironique continue de mettre en rapport le travailleur et la bête, qu'elle tourne en ridicule l'interprétation fataliste du sort des travailleurs et désigne, à travers la complicité de la bête à son propre destin, celle que les patrons et les tenants du statu quo prêtent à l'ouvrier, «responsable de sa misère », comme le dit encore Mauler.

Le matérialisme dialectique qui informe les films d'Eisenstein et les pièces de Brecht interdit évidemment de cultiver univoquement l'identification du travailleur à la bête, et l'animalisation chez le dramaturge caractérise davantage le patron (par la «gueule» de son patronyme) que l'ouvrier. Mais le rapprochement des travailleurs exploités du cochon fut si tenace depuis Sinclair qu'on peut penser qu'il est encore à l'œuvre dans le renversement identificatoire, présenté comme hallucinatoire, avec lequel Georges Orwell conclut sa critique du régime soviétique dans Animal Farm (La ferme des animaux, 1945). Orwell décrit comment les dirigeants de la ferme (les cochons, ces mêmes cochons qui avaient commencé leur mandat en donnant une sépulture aux jambons trouvés dans

29. Bertolt Brecht, Sainte Jeanne des abattoirs, p. 25-26.

30. Bertolt Brecht, Sainte Jeanne des abattoirs, p. 9.

31. Bertolt Brecht, Sainte Jeanne des abattoirs, p. 72. 
la cuisine des humains ${ }^{32}$ ) se métamorphosent au moment où, dans cette même cuisine où ils sont maintenant attablés avec des dirigeants humains, se révèle le caractère irréversible de leur corruption :

But as the animals outside gazed at the scene, it seemed to them that some strange thing was happening. What was it that had altered in the faces of the pigs. Clover's old dim eyes flitted from one face to another. Some of them had five chins, some had four, some had three. But what was it that seemed to be melting and changing? [...] No question now what had happened to the faces of the pigs. The creatures outside looked from pig to man, and from man to pig, and from pig to man again; but already it was impossible to say which was which ${ }^{33}$.

$\mathrm{Si}$, chez Brecht, le travailleur est absent de la description enthousiaste de la machine vers laquelle se précipite le cochon, ailleurs dans la pièce, on apprend que le travail, littéralement, le découpe : l'ouvrier Gloomb a perdu «la main » dans la découpeuse ${ }^{34}$. Mais à ce travailleur morcelé, s'ajoute un autre cas de figure de l'accident de travail, beaucoup plus longuement élaboré et autour duquel se cristallise un second topos, issu lui aussi du roman de Sinclair. Plutôt que le morcèlement partagé de la bête et du travailleur, ce topos va emblématiser à la fois la disparition du travailleur et la voracité du système, celle-ci déjà pointée par le patronyme de Mauler, la mâchoire, comme elle l'avait été dans la représentation de la gueule ouverte de Moloch dans Metropolis de Lang ou dans la machine dentée de La ligne générale d'Eisenstein. Il est inspiré du chapitre neuf de The Jungle qui rassemble un catalogue des horreurs dantesques de l'industrie de la viande, horreurs que Jurgis commence à découvrir au moment de sa fréquentation de plus en plus assidue du syndicat: inspections frauduleuses qui ne refusent aucune bête, même malade, et ferment les yeux devant la réintroduction des déchets de l'abattage dans la cuisson des produits préparés, énumération des maladies et mutilations propres à chaque tâche spécialisée, autant de blessures qui laissent les travailleurs à la rue, sans recours. Le dernier «secret» révélé par ce chapitre concerne l'écueil qui guette les travailleurs affectés aux grandes salles de cuisson envahies de vapeur: «Their peculiar trouble was that they fell into the vats; and when they were fished out, there was never enough of them left to be worth exhibiting-sometimes they would be overlooked for days, till all but the

32. Georges Orwell, Animal Farm, Paris, Gallimard, coll. «Folio bilingue», 2007, p. 6o-61.

33. Georges Orwell, Animal Farm, p. 268-269.

34. Bertolt Brecht, Sainte Jeanne des abattoirs, p. 29. 
bones of them had gone out to the world as Durham's Pure Leaf Lard ${ }^{35}$.» Dans sa préface de 1946, Upton Beall Sinclair, faisant le point sur les modifications apportées à l'industrie suite au scandale créé par la publication de son ouvrage, revient sur ce passage:

The Jungle appeared and became a sensation overnight, not merely in the United States, but in other countries. President Roosevelt sent for me, heard my story, and turned me over to two commissioners whom he ordered to make an investigation of Stockyards conditions.[...] The commissioners told me that the only point on which they could get no proof was my statement that men had fallen into the lard vats and out to the world as pure leaf lard. Naturally this was a hard matter to prove, since in each case the families had been paid off and shipped to other parts of the world ${ }^{36}$.

Il est difficile de savoir si, durant les quarante années écoulées entre la publication initiale du roman et la rédaction de cette préface, Sinclair eut conscience de la fortune critique et des reprises auxquelles donna lieu sa relation de cet accident non vérifié par les inspecteurs. Après tout, la célèbre scène des Temps modernes dans laquelle le vagabond est happé par la machine et passe entre les rouages de la chaîne, si elle traduit littéralement l'expression selon laquelle le travailleur n'est plus qu'un boulon dans l'engrenage, est aussi une façon de figurer, dans un contrepoint à la scène du repas automatisé, la dévoration du travailleur par la machine. Brecht produit une version encore plus élaborée de ce motif:

Un contremaître: «Un gars d'ici, Luckerniddle, est tombé il y a quatre jours dans la chaudière; nous n'avons pas pu arrêter à temps les machines et, c'est effroyable, il a été entraîné dans la chaîne qui débite le lard en tranches; voici sa veste et sa casquette: prend-les et fais-les disparaître; au vestiaire, elles occupent inutilement un porte-manteau et ça ne fait pas bon effet.[...] Dès que l'usine ouvrira, bien sûr tu auras la place de Luckerniddle. ${ }^{37}$

Mais l'ouvrier garde pour lui-même les effets de Luckerniddle car «le copain Tranche-de-Lard n'en a plus besoin, maintenant qu'il est dans sa boîte, habillé de fer blanc». Slift qui, au même moment, fait visiter l'usine à Jeanne afin qu'elle découvre l'immoralité des miséreux, reconnaît la veste et la casquette et confronte le jeune homme. Devant son aveu et son plaidoyer, il ferme les yeux et lui offre un repas à la cantine à condition qu'il prévienne la femme de Luckerniddle qui traîne devant l'usine à la recherche de son mari.

35. Upton Beall Sinclair, The Jungle, p. 114.

36. Upton Beall Sinclair, The Jungle, 1947, p. ix-x.

37. Bertolt Brecht, Sainte Jeanne des abattoirs, p. 27. 
Slift la croise et lui propose un marché: «Ne cherchez pas à savoir où est passé votre mari, et vous pourrez manger gratuitement à notre cantine, le midi, pendant trois semaines ${ }^{38} »$. La femme résiste puis cède à la faim. Le jeune ouvrier arrive à la cantine arborant les effets de son mari et elle l'entend dire à Slift qu'il les tient d'«un homme qui est tombé dans une chaudière ». Nauséeuse, elle sort mais prévient qu'elle se prévaudra de l'offre dès le lendemain pendant trois semaines. Or, au terme de la démonstration de Slift, Jeanne n'est pas convaincue de l'«immoralité sans bornes » des pauvres et elle lui répond: «Ce que tu m’as montré, ce n'est pas l'immoralité des pauvres: c'est leur pauvreté. »

Si le topos de l'homme dans la machine, voire dans le chaudron, prolonge, au-delà du motif du double morcèlement de la tâche et de l'animal, un rapprochement de la bête et du travailleur en transformant ce dernier en nourriture, en «tranches de lard», il constitue aussi clairement un commentaire sur les rapports de l'homme et de la machine, sur la disparition du travailleur dont la menace pointe à travers la sérialisation et l'automation de la production industrielle. Il n'est plus question de morcèlement mais de dévoration. La machine phagocyte le travailleur, elle en fait sa pâture, elle en fait du pâté.

Sauf, bien sûr, dans une version étayée sur une dénégation du topos qui se retrouve dans Tintin en Amérique. Troisième épisode des aventures du reporter belge, Tintin en Amérique conduit le protagoniste à Chicago afin de combattre les criminels. Le récit principal de l'album, rédigé en 1929-1930, mise probablement sur l'intérêt provoqué par un fait divers resté célèbre: le sensationnel Massacre de la Saint-Valentin. L'album regroupe autour de l'industrie de la viande toute une série de lieux communs présentés de manière un peu farfelue. En premier lieu, on retrouve le lien avec l'industrie automobile: les vieilles automobiles sont converties en boîtes de conserve et celles-ci sont à leur tour recyclées en voitures sport. Il y est bizarrement question d'une grève des travailleurs censés avoir arrêté les machines mais ceux-ci s'avèrent plutôt des «fournisseurs » qui réagissent, non à la baisse des salaires, mais à la baisse des prix offerts pour les chiens, les chats et les rats servant à la fabrication du pâté de lièvre: la référence remonte moins à Sinclair qu'à Daumier, et notamment à la célèbre gravure où un chien sert d'ingrédient principal au saucisson de Lyon telle qu'on le cuisine artisanalement 
à Paris. Mais cette reprise véhicule néanmoins ici l'idée de l'insalubrité de la viande produite en industrie 39 .

Parmi les moyens redoutables - et compliqués - mis en œuvre par les criminels pour se débarrasser du justicier Tintin, figure une invitation anonyme à visiter les usines de conserves Slift. Le patronyme de Slift se retrouve étonnamment chez Brecht et chez Hergé. Il s'agit probablement dans les deux cas d'une dérive à partir de Swift. Gustavus F. Swift (1839-1903) fut, avec Phillip D. Armour, un des deux grands industriels de la viande durant le dernier quart du $19^{\mathrm{e}}$ siècle. Son ascension est en partie tributaire du fait qu'il fut un des premiers à faire usage de wagons réfrigérés. En 1902, Armour et Swift établirent un cartel de la viande, la National Packing Company. Il n'est donc pas étonnant que Brecht ait cherché à faire allusion à cette figure. Toutefois, Tintin en Amérique n’appartient pas à la série des ouvrages pour lesquels Hergé se soit minutieusement documenté, ainsi qu'il le fit à partir du Lotus Bleu (1936). Les deux seules sources qu'il aurait consultées sont les Scènes de la vie future de Georges Duhamel et un numéro de la revue ultranationaliste Le Crapouillot. Dans ce numéro, un article de Claude Blanchard évoque les abattoirs de Chicago et mentionne un repas pris à la cantine Swift \& cie. Il est donc probable que ce soit à partir de cette référence, et selon un procédé qui lui était familier, qu’Hergé ait forgé le nom de son protagoniste $4^{\circ}$.

Tintin se rend donc aux usines Slift pour être aussitôt précipité dans la machine que le lecteur n'a pas encore entrevue. Or, autour de la transformation même de l'animal en viande, le cynisme entourant la référence à l'automobile et aux fournisseurs est suspendu au profit d'une version radicalement elliptique du processus: un seul bœuf est visible, encore vivant mais un peu perplexe, au

39. Notons toutefois qu'Hergé prend bien soin d'exonérer le propriétaire des usines de toute responsabilité : après avoir fait tomber Tintin dans la machine, le coupable se frotte les mains en songeant: «Tout se même, si les usines Slift se rendaient compte des ingrédients qui entrent dans la fabrication de leurs conserves ». Il faut donc en déduire que le «chef» qui ordonne de faire disparaître Tintin dans la machine n'est pas le propriétaire des usines.

40. Sur Gustav Swift, voir Siegfried Giedion, Mechanization Takes Command, p. 222228 et la biographie de Swift que fit paraître son fils en 1927: Louis F. Swift, The Yankee and the Yards, the Biography of Gustavus Franklin Swift, Chicago et New York, A.W. Shaw Company, 1927. Pour les sources d'Hergé sur Tintin en Amérique, voir Georges Duhamel, Scènes de la vie future, Paris, Mercure de France, 1930 et Claude Blanchard «L'Amérique et les Américains », Le Crapouillot, octobre 1930, p. 398. Sur Hergé et Le Crapouillot, voir Jean-Marie Apostolidès, Les métamorphoses de Tintin, Paris, Seghers, 1984, p. 30-33. 
moment où il s'apprête à disparaître dans la chaîne automatisée. Dès la vignette suivante, des blocs de corned-beef surgissent à l'autre bout du tapis et celui-ci est flanqué d'un robinet qui déverse un chapelet de saucisses dans un chaudron. Le texte de la première vignette (avec le bœuf) se lit comme suit: «Vous voyez cette énorme machine? Eh bien! Les bœufs arrivent par ici, sur un tapis roulant, à la queue leu leu [...].» Dans la suivante, avec le corned-beef et les saucisses, le même guide déclare: «[...] et ils en sortent de l'autre côté, sous forme de cornedbeef, de saucisses, de graisse à frites [...] Tout se fait automatiquement ${ }^{41}$.» Il est vrai que, malgré les remarques de Giedion sur la nécessité d'un tueur humain, la machine ici paraît accomplir seule le travail de transformation, et le bœuf placide d'Hergé collabore moins à son destin que le cochon courant vers sa fin chez Brecht. Sur ces deux vignettes juxtaposées, aucun corps n'est découpé si ce n'est celui de l'admoniteur. En effet, si le dessin ne laisse voir aucune des violentes opérations subies par l'animal, le corps du guide, lui, est découpé par le cadrage de la vignette et se réduit à un bras qui montre la scène. Avant de précipiter son visiteur dans la machine, le bandit-cicerone lui confie : «Si vous tombiez là-dedans, vous seriez aussitôt broyé par les énormes malaxeurs que vous voyez là sous vos pieds $[\ldots]^{42}$. La barrière cède et Tintin est aussitôt précipité dès la vignette suivante mais la machine demeure invisible: on ne la voit qu'ensuite, avec son gros rouage noir et denté, alors que Tintin a déjà disparu dans un «Platch »43 qui, seul, trahit sa présence dans le chaudron! Mais Tintin ne sera pas transformé en pâté car voilà qu'il y a grève et que les machines ont été arrêtées à l'insu du guide-malfaiteur. On voit plutôt Tintin et Milou sortir salis, mais indemnes, de la machine. "Si cette machine ne s'était pas arrêtée tout de suite, déclare Tintin, nous sortions d'ici sous forme de corned-beef ${ }^{44}$.» Or justement, Tintin et Milou, tous deux à quatre pattes sur le tapis, sont entourés de blocs de viande qui indiquent éloquemment que la machine a continué de produire mais en les épargnant miraculeusement. De plus, leur surgissement de quadrupèdes ahuris annule en quelque sorte la vignette de pâtés et de saucisses qui succédait à la présentation du bœuf vivant et il restaure dans l'image la forme animale de la bête invisiblement abattue et reconfigurée par la machine, occultant ainsi la tuerie et la transformation dont nous pouvons néanmoins constater les effets dans les blocs géométriques et standardisés qui accompagnent Tintin et Milou.

41. Hergé, Tintin en Amérique, p. 53.

42. Hergé, Tintin en Amérique, p. 53.

43. Hergé, Tintin en Amérique, p. 53.

44. Hergé, Tintin en Amérique, p. 54. 
Cette symétrie est une des procédures habituelles par lesquelles Hergé ré-établit l'ordre dans le monde de Tintin ${ }^{45}$. Les vignettes analysées ci-dessus participent d'une autre manière encore à cette stratégie en équilibrant «cet univers carnavalesque où hommes et animaux échangent leurs attributs $4^{46} »$ : cette double tendance se fait singulièrement insistante dans la vignette où le couple Tintin/Milou sort de la machine: Tintin y est dessiné à quatre pattes et Milou, comme c'est souvent son habitude, surtout dans les premiers albums, prend la parole. Cet échange neutralise d'autant plus efficacement la vignette du bœuf que, selon Jean-Marie Apostolidès, le «bestiaire poétique» dans Tintin esquisse une hiérarchie des bêtes établie en fonction de leur bonté ou de leur méchanceté, hiérarchie où les bovidés occupent le degré zéro de la sympathie accordée ${ }^{47}$. Il est absolument significatif, dans le contexte idéologique qui détermine l'œuvre d'Hergé pendant l'entre-deux-guerres, que la réapparition de Tintin se fasse à travers une telle animalisation : c'est dire qu'il paraît plus urgent, surtout pour un jeune public impressionnable, de rétablir un lien avec la bête dont la mise à mort est occultée qu'il ne s'agit de sauver une quelconque figure du travailleur que bien sûr Tintin n'incarne pas ici, contrairement aux instances du topos identifiées chez Sinclair, Brecht ou Chaplin.

Réchappé de la machine, Milou demande: «Et ça arrive souvent des accidents comme ça $4^{8}$ ? " La remarque indique une conscience, chez le dessinateur du moins, du caractère emprunté du motif de l'accident de travail. Or, Hergé n’a très certainement pas lu Sinclair et le topos du travailleur dans la machine lui est parvenu à travers Le Crapouillot et à travers l'article de Claude Blanchard où ce dernier, suite à une visite des abattoirs, s'imagine tombant dans la machine, transformé en «viande de boucherie et saigné par un bœuf ${ }^{49}$ ». Apostolidès voit dans l'écho qu'Hergé donne à ce passage un exemple supplémentaire de ce qu'«un fantasme de dévoration parcourt l'aventure américaine

45. Jean-Marie Apostolidès, Les métamorphoses de Tintin, p. 69.

46. Jean-Marie Apostolidès, Les métamorphoses de Tintin, p. 68.

47. Cette position est pour ainsi dire moderne, compte tenu du fait que depuis l'Antiquité, le débat éthique portant sur le droit de l'homme à tuer des bêtes a longtemps buté sur le capital de sympathie attaché au bœuf, un animal domestiqué, jamais agressif envers l'homme, et qui, de plus, l'aide à labourer ses champs. Sur ces débats, voir Elisabeth de Fontenay, Le silence des bêtes, Paris, Fayard, 1998, p. 49-198. Sur la hiérarchie animale dans Tintin, voir Jean-Marie Apostolidès, Les métamorphoses de Tintin, p. 62-63.

48. Hergé, Tintin en Amérique, p. 54.

49. Claude Blanchard, «L'Amérique et les Américains», p. 399-401. 
de Tintin qui craint d'être broyé dans les engrenages du modernisme ${ }^{5}$ ». De même que Tintin au Pays des Soviets (1930), le premier album de Tintin depuis retiré des œuvres complètes d'Hergé, s'était inspiré d'une littérature anticommuniste, les aventures de Tintin en Amérique reposent sur une documentation fortement anti-américaniste, inspirée par les Scènes de la vie future (1930) de Georges Duhamel et par sa dénonciation d'«une civilisation technicienne et matérialiste en train de conquérir le Vieux-Monde ${ }^{51}$ ». Chez Sinclair, une fois les travailleurs inconnus tombés dans la marmite, il n'y avait plus «rien d'eux à exposer» et ils repartaient «dans le monde» sous forme de Durham Pure Leaf Lard; chez Brecht, le malheureux Luckerniddle devenu Tranche-de-lard se retrouvait « expédié aux quatre coins du monde » dans sa boîte de fer blanc. Mais, accomplie sur quatre pattes, la miraculeuse réapparition du chaste et angélique «globe-trotter» sur le tapis magique de l'automation n'incarne en rien la restitution d'un travailleur déjà disparu, elle manifeste plutôt l'enracinement d'Hergé et de son protagoniste dans les valeurs traditionnelles, catholiques, nationalistes et royalistes de la Vieille Europe qu'il croit menacée par la technique. Le topos du travailleur dévoré par la machine et converti en nourriture, pourtant encore étayé sur un anticapitalisme, vient de changer de camp idéologique.

Post-scriptum : serrant la main de son guide dont il commence à soupçonner la malfaisance, Tintin quitte les usines Slift au volant d'une grosse voiture américaine.

L'auteure remercie le Stirling and Francine Clark Art Research Institute de l'avoir accueillie à l'automne 2007 dans le cadre de cette recherche. Des remerciements également pour leur précieuse collaboration à Ada Ackerman, Émilie Lesage, Eduardo Ralickas, André Habib, Maria Stravinaki et Éric Michaud, ainsi qu'aux doctorants du séminaire Viande et modernité offert à l'automne 2008 au département d'histoire de l'art et d'études cinématographiques de l'Université de Montréal. 